\title{
Delayed fatherhood
}

\author{
Gerald Lawson, ${ }^{1}$ Richard Fletcher ${ }^{2}$
}

\begin{abstract}
${ }^{1}$ Former Consultant Obstetrician and Gynaecologist, John Hunter Hospital, Newcastle, New South Wales, Australia

${ }^{2}$ Senior Lecturer, Faculty of Health and Medicine, Family Action Centre, University of Newcastle, Newcastle, New South Wales, Australia
\end{abstract}

\section{Correspondence to}

Dr Gerald Lawson, 35 Hebburn Street, Hamilton, NSW 2303,

Australia;

gwl8491@hotmail.com

Received 18 December 2013

Revised 31 March 2014

Accepted 24 May 2014

Published Online First

23 June 2014

\section{CrossMark}

\author{
To cite: Lawson $\mathrm{G}$, \\ Fletcher R. J Fam Plann \\ Reprod Health Care \\ 2014:40:283-288.
}

\begin{abstract}
Birth data from developed countries indicates that the average paternal age is increasing. As the trend to older fatherhood has become established, concerns have been raised that this may be linked to adverse outcomes, such as pregnancy complications, congenital anomalies, and long-term health implications for the child. Since the sperm of older fathers may be impaired due to the general effects of ageing, their offspring may be at risk due to defects in sperm quality at conception. A literature search was performed to identify pregnancy complications, fetal anomalies and health issues for the child when the father is in an older age bracket. Evidence for impairment in the sperm and genetic material of older fathers was reviewed. With an older father, there is evidence of an increase in stillbirths and a slightly increased risk of autism, bipolar disorder and schizophrenia in the offspring later in life. The increased risk of achondroplasia has long been recognised. For the mother, there is an increased rate of Caesarean section. Investigations of other possible adverse outcomes have produced mixed findings. Further robust and longitudinal studies are needed to clarify these issues.
\end{abstract}

\section{BACKGROUND}

Statistics from developed countries indicate that the average paternal age is increasing. In England and Wales, the mean age of fathers increased from 29.2 years in 1980 to 32.6 in $2011 .^{1}$ In Norway, the average age of first-time fathers was 26.2 years between 1966 and 1970; in 2009 it was 30.9 years. $^{2}$ Similar trends are reported from Germany ${ }^{3}$ and the USA ${ }^{4}$.

A confounding factor in many of the writings on this issue is the parallel increase in maternal age; older men having their first child are often partnered with a woman also of relatively advanced age. For example, in Australia, the average maternal age in 1971 was 25.4 years; by 2012 it was 30.7 years. $^{5}$ However, not all studies on the paternal age effect make allowance for the

\section{Key message points}

There is an increase in stillbirths when the father is older.

- There is an increased risk of autism, schizophrenia and bipolar disorder when the father is older.

- Despite the above mentioned concerns, the risk to the offspring is low.

increase in maternal age, or for other confounding factors such as sex of the child, the socioeconomic status of the parents, their alcohol and cigarette intake, and a family history of psychosis.

In addition, few studies look at the background health profile of the older father. A Norwegian study ${ }^{2}$ examined this issue in 14832 first-time fathers, and reported that the older men "were more likely to be overweight or obese, to be smokers, and to consume alcohol more frequently than a comparison group".

\section{METHODS}

A search for publications in English dated between 1990 and 2013 was undertaken in the following databases: the Cochrane Library, PubMed, Embase, Scopus and the European Association of Grey Literature. The key words used were: 'delayed paternity', 'paternal age effect', 'ageing sperm', 'pregnancy complications' and 'health issues for the child'. This is a rapidly expanding field. In PubMed alone, under 'paternal age effect' there were 57 new references recorded in 2013. References that were excluded were publications on infertility, reproduction success rates, multiple pregnancies, observational studies, case reports, postbirth paternal health issues, animal studies, and studies where no adjustment was made for maternal age.

A limitation was that complete information on paternal age was not always available. In a review of the 2007 US 
nationwide birth data ${ }^{4}$ it was found that $14 \%$ of the 4316233 deliveries did not have details on the paternal age.

\section{SPERM QUALITY AND AGEING}

The ageing process leads to changes in the cells in the testes that parallel changes in all other parts of the body. However, studies on semen characteristics in the ageing male, in particular sperm morphology, do not provide a uniform picture. A report on 792 American males ${ }^{6}$ undergoing in vitro fertilisation or intrauterine insemination in cases of female infertility only, compared the characteristics of semen in men aged 51-60 years to men aged 20-30 years. The authors reported that the mean percentage of normal sperm morphology in men aged 20-30 years was $69.7 \%$, but this fell to $53.4 \%$ in the older age group. By contrast, a review of sperm characteristics ${ }^{7}$ from fertility units in five countries (USA, France, Spain, Brazil and Egypt) involved in assisted reproductive technology noted a decrease in the percentage of normal morphology with advancing age in only one of six units.

The quality of the genetic material carried in the sperm may also suffer the effects of ageing. Because male germ cells divide continuously, with many more mitotic divisions than the oocytes, the possibility of transcription errors among fathers is greater. ${ }^{8}$ Sperm are produced continuously throughout life. Sperm stem cells divide 30 times before puberty, and from then on every 16 days. By the age of 40 years, a sperm will have gone through 610 cell divisions; at age 50 years, it will have gone through 840 divisions, each time with the possibility of DNA errors in the process, with an increased likelihood of a mutation.

A recent study from Iceland ${ }^{9}$ provided evidence of increased mutations among older fathers. By sequencing the entire genome pattern of 78 parents and their children, the number of mutations passed on to their offspring by the mothers and fathers could be compared. Fathers passed on many times more new mutations than did mothers. The mutation rate was linked to the age of the father at conception, with an increase of about two mutations per year.

\section{COMPLICATIONS IN THE PREGNANCY}

A number of adverse outcomes such as miscarriages, stillbirths, preterm births and Caesarean sections have been studied to assess the paternal age effect.

The early loss of a fetus occurs in approximately $10-15 \%$ of pregnant women. Many of these pregnancy losses are thought to be due to chromosomal errors in the fetus. A study of 5121 pregnant Californian women ${ }^{10}$ found that the adjusted hazard ratio (HR) for miscarriage where the father was aged 35 years or more, compared with less than 35 years, was 1.27 [95\% confidence interval (CI) 1.00-1.61], adjusted for maternal age. However, a different experience was reported for European women, ${ }^{11}$ where mixed outcomes were reported for three age brackets: "If the woman was 20-29 years old, the risk of miscarriage was not significantly high, whatever the age of the man. If the woman was 30-34 years old, the risk of miscarriage was higher if the man was greater than 40 years. If the woman was greater than 35 years, the risk of miscarriage increased, whatever the age of the man". Accordingly, the authors argued that the risk of miscarriage was a "couple's business", rather than just attributable to the mother or father.

Stillbirths may also be linked to an older father, a possibility that was first raised in $1939 .{ }^{12}$ More recently, a Missouri study ${ }^{13}$ reviewed the outcome of 755334 deliveries between 1989 and 2005. There were 49419 (6.5\%) fathers aged 40 years or over. The authors reported that, after adjusting for maternal age, compared with infants born to younger fathers (25-29 years), those born to fathers aged $40-45$ years had a $24 \%$ increased risk of stillbirth [odds ratio (OR) 1.24; 95\% CI 1.04-1.47]. A Danish study ${ }^{14}$ analysed 23821 pregnancies, of which 146 ended in stillbirth after 20 weeks' gestation. It reported that pregnancies fathered by a man aged 50 years or older had almost twice the risk of ending in fetal loss, compared with pregnancies by younger fathers (HR 1.88; 95\% CI 0.93-3.82), after adjustment for maternal age. An Italian study ${ }^{15}$ reviewed the outcome of 3619647 deliveries between 1990 and 1996, and analysed fetal deaths from 26 weeks. It found that paternal age over the age of 40 years increased the risk of stillbirth in mothers over 35 years of age, although this was modified if the mother had had a "high educational level", thought to reflect better socioeconomic conditions. The consistency of these findings indicates a risk of increased stillbirth with an older father.

The evidence that advanced paternal age can be linked to a preterm birth is contradictory. An American study of 2509012 live births of married women aged 20-34 years from white, non-Hispanic first-time mothers ${ }^{16}$ did not identify a link between older paternal age and early preterm delivery. By contrast, the Missouri study quoted above ${ }^{13}$ claimed that with paternal age of 45 years or more, there was a $13 \%$ increased risk of preterm delivery between 33 and 37 weeks (OR 1.13; $95 \%$ CI $1.05-1.22$ ), and a $29 \%$ risk of very preterm delivery, less than 33 weeks, compared to fathers aged 25-29 years (OR 1.29; 95\% CI 1.15-1.44).

An increase in the Caesarean section rate with increasing paternal age has been consistently reported. A Taiwanese report ${ }^{17}$ indicated that for a mother aged 20-29 years, the Caesarean rate was $27.2 \%$ when the father was aged 20-29 years; but this increased to $35.8 \%$ if the father was over 40 years old. A study from New Jersey ${ }^{18}$ reported that, after adjusting for maternal age, the Caesarean section rate for infants of Caucasian fathers aged 20-29, 30-39 and 40+ years was $21.1 \%, 26.7 \%$ and $31.8 \%$, respectively. 
The prevalence of pre-eclampsia (or pregnancy-induced hypertension) was reported in an Israeli study ${ }^{19}$ as being lowest when the father was aged 25-34 years. There was an increased risk of the disorder with fathers aged over 34 years, but the risk was also slightly elevated in younger fathers, aged between 20 and 24 years. Nonetheless, the study found that the overall contribution to the disorder by the father was "modest" compared to the effect of maternal age. The Missouri study mentioned above ${ }^{13}$ also noted that it was both the young fathers aged under 20 years, and the older fathers over 45 years, who made a slight contribution to the incidence of pre-eclampsia.

\section{CONGENITAL ANOMALIES IN THE OFFSPRING}

Given the plausibility of increased mutations in the genetic material of older fathers, congenital birth defects would be expected to show a significant increase in line with paternal age. Since records of birth defects are available for large populations, links between paternal age and even rare congenital anomalies should be apparent.

An analysis of the 5213248 American deliveries between 1999 and $2000^{20}$ noted that there were $77514(1.5 \%)$ offspring with birth defects. Compared to the father's age group of 25-29 years, infants born to fathers aged $50+$ years had a $15 \%$ increase in defects (OR 1.15; 95\% CI 1.06-1.24). However, infants born to mothers aged $45+$ years had a 2.2 -fold increase in defects. In other words, the mother's advancing age was a stronger factor in contributing to birth defects than the age of the father. However, the authors concluded that increased paternal age was associated with a slightly increased risk of a number of rare disorders, namely heart defects, tracheo-oesophageal fistula, oesophageal atresia and some chromosomal disorders.

A review of data from the American National Birth Defects Prevention Study ${ }^{21}$ concerning infants born between 1997 and 2004 looked at 50 types of congenital defect. It noted increased risk with increasing paternal age for diaphragmatic hernia, cleft palate, right heart ventricular outflow obstruction and pulmonary vein stenosis. Again, these are rare conditions.

By contrast, a Norwegian study ${ }^{22}$ of 1869388 births reported no "consistent evidence" of increased risk for either paternal or maternal age.

A Danish study ${ }^{23}$ reported no association between paternal age and the overall prevalence of congenital malformations, but it did report an increase in some specific conditions, such as Down's syndrome, malformations of extremities, and syndromes of multiple systems.

A major congenital anomaly, Down's syndrome (trisomy 21), has been linked to increasing maternal age since it was first described by British geneticist Lionel Penrose in $1933,{ }^{24}$ but links with paternal age have been unclear. The Danish study mentioned above $^{23}$ did report an increase in trisomy 21 with increased paternal age. However, an analysis of cases of trisomy 21 identified from the Texas Birth Defects Registry from 1999 to $2007^{25}$ found an increased rate for fathers aged over 40 years, and also for fathers aged 20-24 years, but only when the mother was aged over 35 years. An examination of 3419 trisomy 21 cases from the New York congenital malformations registry from 1983 to 1997 claimed that there was a paternal contribution, but only if both the mother and the father were both aged over 35 years. ${ }^{26}$

Contradictory results have also been reported for multiple sclerosis and retinoblastoma. An analysis of the Swedish Inpatient Register ${ }^{27}$ identified 4443 patients with multiple sclerosis, and reported that the risk increased with the father's age, but not the mother's. For 51-55-year-old fathers, the OR was 2.0 (95\% CI 1.35-2.96) compared to fathers aged 21-25 years. By contrast, a study from a Canadian database ${ }^{28}$ found no such link in a review of 5681 multiple sclerosis cases.

Retinoblastoma is a condition that affects 1 in 15000 births, and is the commonest inherited malignancy in childhood. A study of 187 cases of bilateral retinoblastomas in children aged under 6 years, identified from the Californian Cancer Registry, ${ }^{29}$ reported that the disorder was associated with paternal age for fathers over 35 years (crude OR 1.73; 95\% CI 1.202.47). However, a Swedish study, ${ }^{30}$ reviewing 226 cases of retinoblastoma in children aged under 5 years, did not find such a link to paternal age.

\section{LONG-TERM HEALTH COMPLICATIONS}

\section{FOR THE CHILD}

Increasing paternal age at conception has been considered as a possible contributor to a number of serious mental illnesses such as autism, bipolar disorder and schizophrenia. The aetiology of these conditions remains elusive, and there is no one single cause. A number of contributing factors have been identified, one of which appears to be an older father. Other risk factors are preterm birth, being the first-born child, a low birth weight less than $2500 \mathrm{~g}$, being small for gestational age at birth, being born with a low Apgar score, and breech delivery. However, none of the known individual risk factors are present in the majority of cases, so our understanding of the causes of these conditions remains incomplete. It is postulated that de novo chromosome mutations in the sperm of older men may be a reason for their association with these disorders. ${ }^{31}$

The prevalence of autism has increased in recent decades. It is thought to occur in $10-20$ per 10000 individuals. Controversy remains as to whether this is a true rise or whether it is because of a change in diagnostic criteria, or a shift to diagnosis at an earlier age. 
A report on 818 Danish children with autism born between 1984 and $1998^{32}$ found that the highest risk was for individuals who had a sibling diagnosed with autism. However, this study also reported that the risk of autism increased with paternal age, with the relative risk in the 40-44-year age bracket being 1.61 (95\% CI 1.19-2.18) compared to fathers aged 25-29 years. A similar result was obtained in an analysis of data on Israelis with autism who were born in six consecutive years during the $1980 \mathrm{~s} ;{ }^{31}$ the adjusted OR was 5.75 (95\% CI 2.65-12.46) when the father was aged 40-49 years, compared to fathers aged under 30 years.

An examination of Californian birth records between 1990 and $1999^{33}$ resulted in a more complicated picture of the connection between paternal age and autism. Out of 4947935 births, the researchers identified 12159 individuals with autism (246 per $100000)$. They reported that advancing maternal age increased the risk for autism, regardless of the paternal age. Compared with mothers aged 25-29 years, the adjusted OR for mothers aged 40 years and over was 1.51 (95\% CI 1.35-1.70). In contrast, advancing paternal age was associated with the risk of autism only when the mother was under 30 years (OR 1.59; $95 \%$ CI 1.37-1.85). Other studies from the USA ${ }^{34} 35$ suggest that both increasing paternal and maternal age are independently associated with autism in the offspring. An Australian study ${ }^{36}$ also found that autism cases had significantly older parents, though the paternal age factor was statistically weaker than the maternal age. By contrast, a study from Denmark ${ }^{37}$ did not find a statistically significant association with either increasing maternal or paternal age.

Complicating the picture is that amongst the studies of autistic patients, there is "considerable variation in methods, sample size, variable selection, analyses, data quality and control groups". ${ }^{36}$ For example, the studies from the USA often include Caucasians, black Americans, Hispanics and Asians, whereas those from other countries generally assess more homogenous groups. Nonetheless, advancing paternal age appears to be one of several contributing factors in the development of autism.

Studies of bipolar disorder have also reported contradictory results. In Sweden since 1947, every citizen has had a personal identification number, allowing precise longitudinal data. From a population of 7328100 , there were 13428 patients identified with bipolar disorder. ${ }^{38}$ The offspring of men aged 55 years and over were more likely to be diagnosed as having bipolar disorder than the offspring of men aged 20-24 years (OR 1.37; 95\% CI 1.02-1.84). The maternal age effect was less pronounced. By contrast, a Californian study ${ }^{39}$ comparing 94 bipolar disorder cases with 746 controls found no increased risk with paternal ageing.

Paternal age has been examined in studies of schizophrenia among Israeli, American and Swedish populations. An Israeli study investigated the background of 658 persons admitted to psychiatric facilities in Jerusalem prior to 1998 with a diagnosis of schizophrenia. ${ }^{40}$ The prevalence increased progressively with advancing paternal age. Compared with offspring of fathers aged less than 25 years, the relative risk of schizophrenia was 2.02 (95\% CI 1.17$3.51)$ and 2.96 (95\% CI 1.60-5.47) in offspring of men aged 45-49 and 50 years or more, respectively.

In a study of schizophrenia diagnosed in 25025 children born in three American cities (Boston, Providence and Philadelphia) between 1959 and $1966,{ }^{41}$ together with a literature review of nine other similar studies, the authors found that the fathers were slightly but not significantly older. They argued that compared with other risk factors for schizophrenia, advanced paternal age appears to be "intermediate in magnitude", and only a risk factor among offspring of fathers aged 55 years and over.

A Swedish study ${ }^{42}$ reviewed the files of 754330 children born between 1973 and 1980. It identified $639(0.08 \%)$ individuals who had a diagnosis of schizophrenia. The authors reported that there was an increased risk with older fathers, and that the HR for each 10-year increase in paternal age was 1.47 (95\% CI 1.23-1.76).

\section{ACHONDROPLASIA}

Achondroplasia is a rare condition that has been consistently linked with paternal ageing. It was the first disorder to be so identified by Wilhelm Weinberg in 1912. ${ }^{43}$ The German physician noticed that it often presented in the last-born child of large families. His findings were confirmed by other studies in the mid-20th century by Mörch in $1941^{44}$ and Penrose in $1955 .^{45}$

An American study in 1970 of 148 achondroplastic $\mathrm{dwarfs}^{46}$ reported that the average paternal age for the disorder was 6.2 years older than fathers of normal children in the same population. A 1995 study ${ }^{47}$ reported that the mean paternal age for Italian children with achondroplasia was 36.3 vs 31.7 years for matched controls; for Latin American children, the mean paternal age was reported as 37.19 vs 27.53 years for controls. The disorder is thought to be due to increased mutation frequency at the fibroblast growth factor receptor 3 (FGFR3) gene, ${ }^{48}$ though a recent theory has suggested that it may be due to 'selfish' mutant spermatogonial stem cells. ${ }^{49}$

\section{DISCUSSION}

As a man ages and his general physiological fitness declines, it seems reasonable to ask if the quality of the genetic material that he passes on to his children through the mother is compromised. Having clear evidence of adverse effects from an increase in paternal age would assist clinicians advising parents and help underpin public health approaches to support 
children's healthy birth and development. However, the challenge in reversing the trend of ageing paternity is considerable. A Norwegian group ${ }^{2}$ has suggested incentives, such as "facilitation of parental leave and improved financial conditions for couples to conceive a few years earlier than is usual in modern societies of today".

While there seems to be sufficient evidence to confirm that an older father's chromosomal material will carry increased mutations, the effects of these on his partner and on his children's future is much less clear. In the case of congenital anomalies, which represent the most immediate measure of impairment due to the father's genetic load, there is mixed evidence of an overall increase of such conditions linked to the father's age at conception. With the exception of achondroplasia, the evidence is inconsistent, or points to the effects of older fathers and older mothers combining to produce the negative outcome.

Studies of long-term serious outcomes for children, such as autism or schizophrenia, are suggestive but do not clearly demonstrate that the age of the father is a definitive factor. In the words of two German researchers, ${ }^{3}$ "even if the genetic risk for progeny from older fathers is slightly increased, the risk to the individual is low".

It should also be recognised that although there has been an increase in research on the paternal age effect, much of the research is correlational and few longitudinal studies specifically designed to answer questions relating to paternal age have been conducted. Obviously, further robust and longitudinal studies on these issues are needed, allowing for confounding factors such as the mother's age and the background of both parents.

It should also be acknowledged that there are significant benefits for a child born to an older father, in that the father may have become financially established, and be more emotionally mature to take on the role of fatherhood. Against this there is the possibility that the father may have his own health problems, and that he might die before the child reaches adulthood.

On a more philosophical note, the question of an optimum time to father a child has been addressed by $\mathrm{Liu}^{50}$ who suggested that "it is not unreasonable to propose that the decade between 30 and 40 years of age is the optimal time for fathers to have a child, balancing both intelligence and health of the children".

Acknowledgements The authors wish to thank Dr Ken Maclean for reviewing an early draft of this article.

Competing interests None.

Provenance and peer review Not commissioned; externally peer reviewed.

\section{REFERENCES}

1 Office for National Statistics (UK). Patterns of fatherhood in England and Wales, 1964-2007. Popul Trends

2009;136:103-107.
2 Nilsen $\mathrm{AB}$, Waldenstrom U, Rasmussen S, et al. Characteristics of first-time fathers of advanced age: a Norwegian population-based study. BMC Pregnancy Childbirth 2013;13:29.

3 Kuhnert B, Nieschlag E. Reproductive functions of the ageing male. Hum Reprod Update 2004;10:327-339.

4 Martin J, Hamilton B, Sutton P, et al. Births: final data for 2007. Centers for Disease Control and Prevention. Natl Vital Stat Rep 2010;58:1-86.

5 Births Australia, 2009. Australian Bureau of Statistics. 3301.0; November 2010: 1-68.

6 Zavos P, Kaskar K, Correa J, et al. Seminal characteristics and sexual behaviour in men of different age groups: is there an aging effect? Asian J Androl 2006;8:337-341.

7 Dain L, Auslander R, Dirnfeld M. The effect of paternal age on assisted reproduction outcome. Fertil Steril 2011;95:1-8.

8 Crow J. The origins, patterns and implications of human spontaneous mutation. Nat Rev Genet 2000;1:40-47.

9 Kong A, Frigge M, Masson G, et al. Rate of de novo mutations and the importance of father's age to disease risk. Nature 2012;488:471-475.

10 Slama R, Bouyer J, Windham G, et al. Influence of paternal age on the risk of spontaneous abortion. Am J Epidemiol 2005; 161:816-823.

11 de La Rochebrochard E, Thonneau P. Paternal age and maternal age are risk factors for miscarriage; results of a multi-centre European study. Hum Reprod 2002;17:1649-1656.

12 Yerushalmy J. Age of father and survival of offspring. Hum Biol 1939;11:342-356.

13 Alio A, Salihu H, McIntosh C, et al. The effect of paternal age on fetal birth outcomes. Am J Mens Health 2012;6:427-435.

14 Nybo Anderson A, Hansen K, Andersen P, et al. Advanced paternal age and the risk of fetal death: a cohort study. Am J Epidemiol 2004;160:1214-1222.

15 Astolfi P, De Pasquale A, Zonta L. Late paternity and stillbirth risk. Hum Reprod 2004;19:2497-2501.

16 Basso O, Wilcox A. Paternal age and delivery before 32 weeks. Epidemiology 2006;17:475-478.

17 Tang C, Wu M, Liu J, et al. Delayed parenthood and the risk of cesarean delivery - is paternal age an independent risk factor? Birth 2006;33:18-26.

18 Faro R, Santolaya-Forgas J, Canterino J, et al. Paternal age and risk for cesarean delivery. J Matern Fetal Neonatal Med 2012;25:2713-2716.

19 Harlap S, Paltiel O, Deutsch L, et al. Paternal age and pre-eclampsia. Epidemiology 2002;13:660-667.

20 Yang Q, Wen S, Leader A, et al. Paternal age and birth defects: how strong is the association? Hum Reprod 2007;22:696-701.

21 Green R, Devine O, Crider K, et al. Association of paternal age and risk for major congenital anomalies. Ann Epidemiol 2010;20:241-249.

22 Kazaura M, Lie R, Skjaerven R. Paternal age and the risk of birth defects in Norway. Ann Epidemiol 2004;14:566-570.

23 Zhu J, Madsen K, Vestergaard M, et al. Paternal age and congenital malformations. Hum Reprod 2005;20:3173-3177.

24 Penrose L. The relative effects of paternal and maternal age in mongolism. J Genet 1933;27:219-224.

25 Agopian A, Marengo L, Mitchell L. Predictors of trisomy 21 in the offspring of older and younger women. Birth Defects Res A 2012;94:31-35.

26 Fisch H, Hyun G, Golden R, et al. The influence of paternal age on Down syndrome. J Urol 2003;169:2275-2278. 
27 Montgomery S, Lambe M, Olsson T, et al. Parental age, family size and risk of multiple sclerosis. Epidemiology 2004;15:717-723.

28 Ramagopalan S, Dyment D, Guimond C, et al. No effect of parental age on risk of multiple sclerosis: a population-based study. Neuroepidemiology 2010;34:106-109.

29 Heck J, Lombardi C, Meyers T, et al. Perinatal characteristics and retinoblastoma. Cancer Causes Control 2012;23:1567-1575.

30 Yip B, Pawitan Y, Czene K. Parental age and the risk of childhood cancers: a population-based cohort study from Sweden. Int J Epidemiol 2006;35:1495-1503.

31 Reichenberg A, Gross R, Weiser M, et al. Advancing paternal age and autism. Arch Gen Psychiatry 2006;63:1026-1032.

32 Lauritsen M, Pedersen C, Mortensen P. Effects of familial risk factors and place of birth on the risk of autism: a nationwide register-based study. J Child Psychol Psychiatry 2005;46:963-971.

33 Shelton J, Tancredi D, Hetz-Picciotto I. Independent and dependent contributions of advanced maternal and paternal ages to autism risk. Autism Res 2010;3:30-39.

34 Durkin M, Maenner M, Newschaffer C, et al. Advanced paternal age and the risk of autism spectrum disorder. Am J Epidemiol 2008;168:1268-1276.

35 Grether J, Anderson M, Croen L, et al. Risk of autism and increasing maternal and paternal age in a large North American population. Am J Epidemiol 2009;170:1118-1126.

36 Glasson E, Bower C, Petterson B, et al. Perinatal factors and the development of autism: a population study. Arch Gen Psychiatry 2004;61:618-627.

37 Larsson H, Eaton W, Madsen K, et al. Risk factors for autism: perinatal factors, parental psychiatric history and socioeconomic status. Am J Epidemiology 2005;161:916-925.
38 Frans E, Sandin S, Reichenberg A, et al. Advancing paternal age and bipolar disorder. Arch Gen Psychiatry 2008;65:1034-1040.

39 Brown A, Bao Y, McKeague I, et al. Parental age and risk of bipolar disorder in offspring. Psychiatry Res 2013;208:225-231.

40 Malaspina D, Harlap S, Fennig S, et al. Advancing paternal age and the risk of schizophrenia. Arch Gen Psychiatry 2001;58:361-367.

41 Torrey E, Buka S, Cannon T, et al. Paternal age as a risk for schizophrenia: how important is it? Schizophr Res 2009;114:1-5.

42 Sipos A, Rasmussen F, Harrison G, et al. Paternal age and schizophrenia: a population based cohort study. BMJ 2004;329:1070-1073.

43 Weinberg W. Zur Vererbung des Zwergwuchses. Arch Rass Gesantte Biol 1912;9:710-717.

44 Mörch E. Chondrodystrophic Dwarfs in Denmark. Copenhagen, Denmark: Ejnar Munksgaard, 1941.

45 Penrose L. Parental age and mutation. Lancet 1955;2:312-313.

46 Murdoch J, Walker B, Hall J, et al. Achondroplasia - a genetic and statistical survey. Ann Hum Genet 1970;33:227-244.

47 Orioli I, Castilla E, Scarano G, et al. Effect of paternal age in achondroplasia, thanatophoric dysplasia and osteogenesis imperfecta. Am J Med Genet 1995;59:209-217.

48 Bellus G, Hefferon T, Ortiz de Luna R, et al. Achondroplasia is defined by recurrent G380R mutations of FGFR3. Am J Hum Genet 1995;56:368-373.

49 Goriely A, Wilkie A. Paternal age effect mutations and selfish spermatogonial selection. Causes and consequences for human disease. Am J Hum Genet 2012;90:175-200.

50 Liu Y. When is the best age to have a child? Birth 2011;38:276. 